\title{
Genome dimensions control biological and toxicological functions; myth or reality?
}

\author{
Diane C. Wang • Xiangdong Wang
}

Received: 2 July 2018 / Accepted: 9 July 2018 /Published online: 14 July 2018

(C) Springer Nature B.V. 2018

\begin{abstract}
The multidimensional genome offers a new perspective to understand molecular mechanisms of genotoxicity and provide deeper knowledge of how genome organization and reorganization in dimensions can alter cell sensitivity, tolerance, resistance, or toxicity to drugs, whether drugs per se can influence the 3D architecture of the genome directly or indirectly through transcriptional factors, and how we can improve cell sensitivity to drugs through the reorganization of genome and regulation of gene expression. We address roles of $3 \mathrm{D}$ genome organization and reorganization in the pathogenesis and progression of disease by evaluating various methodologies of studying the 3D genome, and in the genome integrity and stability susceptible to chemicals as mechanisms of genotoxicity. We discuss the value of imaging, visualizing, and nuclear proximity ligationbased methods of 3D genome organization to measure spatial proximity and visualize spatial distances between genomic loci. We also list a number of dynamic genome changes during genome function and call for further investigations on the interaction of drugs with genomespecific regulators, key enzymes, or spliceosome.
\end{abstract}

\section{C. Wang $\cdot$ X. Wang}

Zhongshan Hospital Institute of Clinical Science, Zhongshan Hospital, Fudan University Shanghai Medical College, Shanghai, China

\section{C. Wang $\cdot$ X. Wang $(\bowtie)$}

Shanghai Institute of Clinical Bioinformatics, Shanghai, China e-mail: xiangdong.wang@ clintransmed.org
Keywords Multiple dimensions · Genome · Drugs · Toxicity $\cdot$ Regulators

Multidimensional organization of the genome is a new area to be explored to further the understanding of the cell and discovering more applicable elements for clinical diagnosis and therapy. In 1993, McLaren made an obscure proposal and foresaw that gene structure and arrangement of chromosome could be four-dimensional on computer screens in 2000. Although the concept of genome dimensions then is definitely different from the one today, it indicates that genetic scientists believe the complexity of genome function and regulation is associated with or dependent upon the gnome dimension. From back then, there has been increasing evidence that the threedimensional (3D) architecture of the genome plays critical roles in gene expression, signal regulation, cell biology, and organ function. More recently, the four-dimensional (4D) structure of the genome was headlined as the structure and dynamics of genomes in space and time. The 4D Nucleome Project is organizing global effort to furthermore define molecular mechanisms by which the nucleus is organized and function, discover novel approaches of measurements of genome conformation and nuclear organization, and explore the regulatory factors and interactions (Dekker et al. 2017). With the development of biotechnology, the 5D, 6D, and multiple dimensions of the genome will be defined and found to regulate and decide more signaling pathways and additional factors, which contribute to the regulation of genome architecture and reconstructions. The understanding of $3 \mathrm{D}$ or $4 \mathrm{D}$ genome structure will provide deeper knowledge of how genome organization and reorganization in 
dimensions can alter cell sensitivity, tolerance, resistance, or toxicity to drugs, whether drugs per se can influence the 3D architecture of the genome directly or indirectly through transcriptional factors, and how we can improve cell sensitivity to drugs through the reorganization of genome and regulation of gene expression.

It is a challenge to understand how 3D genome organization and reorganization play important roles in the pathogenesis and progression of disease. Li et al. explored the significance of $3 \mathrm{D}$ genome structure in the investigation of cancer and diseases by evaluating various methodologies of studying the 3D genome, e.g., chromosome conformation capture (3C) and 3C-based technologies (Hi-C, ChIA-PET) (2018). Those have the advantage of defining the non-coding genome alterations resulted from chromatin interactions and reorganization in pathological conditions. The microscopy-based methodologies are applied for 3D genome organization and chromatin behaviors. The selection of methods in 3D genome research is highly dependent upon the scientific focus and molecular relationship between 3D genome and transcription, DNA replication, and DNA repair (Dostie and Blanchette 2018). For example, the fluorescence in situ hybridization technique is more suitable for tracing targeted genome regions and observing the $3 \mathrm{D}$ position of short (35-300 kb), specific genomic loci in cancer cells. The 3D genome architecture of multiple myeloma, functional roles, and relationship with genomic variation and gene expression were initially investigated by the integration of $\mathrm{Hi}-\mathrm{C}$, whole-genome sequencing, and RNA-seq (Wu et al. 2017). This is an example of how to optimize the methods to show that copy number variation breakpoints significantly overlap with topologically associating domain boundaries and address the difference between normal and abnormal B cells, e.g., the number and size of topologically associating domain increased and decreased about $20-25 \%$ in disease, respectively. It is also a challenge to achieve optimization of the used methodologies, explain the variations of data generated from different measurements, as well as define the meanings of altered 3D genome organization in the disease. The finding that $20 \%$ of genomic regions switched the chromatin A/B compartment types may have any correlation and association with clinical phenomes, any regulation of clinical trans-omics, or any link with therapeutic responses (Wang 2018).

The genome integrity and stability are susceptible to chemicals, leading to the occurrence of genotoxicity, although effects of drugs on $3 \mathrm{D}$ dimensional genome architectures are not fully understood. Terabayashi and Hanada demonstrated potential association of mutations in DNA repair/damage responses-related genes with clinical representative phenomes in genome instability syndromes, e.g., the high incidence of cancers, short stature, microcephaly, and/or neurological deficiencies (2018). A number of factors, e.g., physical interactions between chromatin regions, structural variability of the genome between cells, or long-range and interchromosomal interactions can affect 3D genome structures and stability. The stability of large centromere clusters with distinct chromosome compositions can vary between cells and between their chromosome identities (Tjong et al. 2016). The chromosome-specific clusters may guide chromosome positioning and stabilize chromatin interactions. Each of 3D chromatin clusters can cross a population of genome structures, either deconvoluted from ensemble-averaged $\mathrm{Hi}-\mathrm{C}$ data or from a collection of single-cell Hi-C data. Dai et al. developed an atlas of stable inter-chromosomal chromatin clusters and the concept of the 'Regulatory Communities,' where most clusters bind with specific regulatory factors and both centromere clustering and transcription factor binding play a critical role in the maintenance of community stability (Dai et al. 2016). The intercellular heterogeneity of the regulatory communities also exists and influences multiple dimensions of genome structures, consequently impacting expression variability. Single-cell dynamic phenotypes and intratumor heterogeneity were also suggested to have a close association with cancer genome instability, evolution, and drug resistance (Wang et al. 2017). Genome organization and stability when faced with genotoxic stress may be associated with the circadian rhythmicity induced by the molecular clock. The cellular response to DNA damage was impacted when the molecular regulatory system was irradiated with $\gamma$-rays at times, leading to alterations of clonogenic cell survival, double-strand break repair kinetics, and TP53 protein levels (Corrà et al. 2017).

Imaging, visualization, and nuclear proximity ligation-based methods of 3D genome organization are suggested as the main strategies to measure the spatial proximity and visualize spatial distances between genomic loci as well as map global 3D genome structures and visualize genome 3D structures. Ma et al. systemically overviewed a number of novel imaging and visualization methods to study 3D genomes in the present issue (2018). The probe labeling and microscopical resolution are the limiting factors to image and 
visualize chromatin interactions and 3D genome architectures. Quinodoz et al. (2018) made a significant development of split-pool recognition of interactions by tag extension to monitor higher-order interactions within the nucleus, including known structures and interchromosomal interactions around the nucleolus and nuclear speckles. This is a global model to demonstrate roles of nuclear bodies in the overall packaging of DNA in the nucleus and investigate both genome-wide structure and interactions with nuclear bodies. The computational model of 3D genome analyses is an important and efficient high-throughput part in order to reconstruct large systems, especially after the integration of artificial intelligence. For example, the manifold-based optimization was proposed for the reconstruction of 3D genome structure from chromosomal contact data with fewer structural violations (Paulsen et al. 2015). The artificial intelligent single-cell model is recently proposed as a single-cell-like system with computerized databases, digitalized informatics of biological elements, and programmed function and signals (Zeng et al. 2018). On the basis of the principle, it is possible to establish an artificial intelligent 3D genome as an optimal system with 3D genome measurements (e.g., Hi-C, imaging, visualization, others), intelligent capacity of analyzing and deep learning, statistical comparisons, and precise interpretation of variations. Such intelligent 3D genome is used as a computational automation-based analysis to standardize the process of data collection, management, and analysis, reduce the degree of sparse and noisy interaction mining, and optimize the non-aggregated structural assessment of genome structure.

3D genome organization and reorganization are more spatial, flexible, and dynamic. For example, chromatin loops usually vary in length and duration, including temporal or persisting loops in the cell cycle. Szalaj and Plewczynski (2018) more systemically address the dynamics of the genome architecture ranging from chromatin loops to the whole genome, cell-to-cell variability, and relationship between genome reorganization with chromosome $\mathrm{X}$ inactivation or cell differentiation. The enhancer interacts with the promoter to dynamically form a variety of complex and cell-type specific networks of interconnected genes, regulating the function of transcriptional factor networks and the spatial clustering of genes and regulatory factors. Those factors can then contribute to the organization and dynamics of genome function and chromosome architecture during the cell cycle. The dynamic and unstable binding of cohesin is responsible for weaker globule boundaries. The dynamic nature and behavior of the chromatin fiber and chromosome movements that contribute to the dynamic reorganization of the genome occurs in response to environmental or developmental stimuli. A large number of dynamic changes occur during genome function, including dynamic reorganization of chromosomes for the movement of centromeres to a telomereproximal microenvironment dynamic interphase chromatin for nuclear territoriality, or dynamic motion of chromatin for the characteristic static snapshot of chromosome architecture (Mizuguchi et al. 2015; Szalaj and Plewczynski 2018). The architecture, dynamics, accessibility, balance stability, and flexibility play important roles in the maintenance of genome integrity and variation for gene expression and regulation by selforganization of active or inactive units in proximity (Knoch et al. 2016). One challenge is to design a clear study on the spatial organization and dynamics of specific loci simultaneously, especially in pathophysiological conditions and during the disease exacerbation.

Deeper understanding of multiple dimensions of genome architecture will provide us with better knowledge of molecular mechanisms by which the genome regulates and controls transcriptional signaling. There are more investigations urgently needed to explore the roles of $3 \mathrm{D}$ or $4 \mathrm{D}$ genome organization and its regulators in the interaction between drugs and cells. It should be questioned whether the cell develops resistance and tolerance to chemotherapy or target drugs through alternations of $3 \mathrm{D}$ genome organization. It is possible that drug toxicity can cause changes of genome dimensions, since drugs may influence gene expression profiles and mature phenotypes of human embryonic stem cells (Kim et al. 2018). Drugs may interact between heterochromatic regions and affect the interconversion between inactive heterochromatic and active euchromatic genomic regions and interactions with lamina, leading to altered organization of conventional and inverted nuclei. It will be helpful to have measures of chromatin-bound RNAs, e.g., nascent transcripts, chromosome-specific dosage, compensation ncRNAs, and genome-wide trans-associated RNAs in drugspecific toxic processes. Studies on multidimensional genome will help develop our understanding of genotoxicity to a new level. We should pay special attention to the interaction of drugs with genomespecific regulators (e.g., CTCF and CTCF-binding elements), key enzymes (e.g., RNA polymerases and 
activation of its C-terminal domain), or spliceosome consisting of small nuclear ribonucleoproteins and other proteins (e.g., heterogeneous nuclear ribonucleoproteins). We also expect to map disease-specific 3D genome organization from nucleosomes, loops, topologically associating domains, compartments, to chromosome territories.

In conclusion, understanding the multidimensional genome will guide us to a new level of understanding molecular mechanisms of genotoxicity and provide deeper knowledge of how genome organization and reorganization in dimensions can alter cell sensitivity, tolerance, resistance, or toxicity to drugs, whether drugs per se can influence the 3D architecture of the genome directly or indirectly through transcriptional factors, and how we can improve cell sensitivity to drugs through the reorganization of genome and regulation of gene expression. We address roles of 3D genome organization and reorganization in the pathogenesis and progression of disease by evaluating various methodologies of studying the 3D genome, and in the genome integrity and stability susceptible to chemicals as mechanisms of genotoxicity. We discuss the value of the imaging, visualizing, and nuclear proximity ligation-based methods of 3D genome organization to measure the spatial proximity and visualize spatial distances between genomic loci. We also list a number of dynamic genome changes during genome function and call for further investigations on the interaction of drugs with genome-specific regulators, key enzymes, or spliceosome.

\section{References}

Corrà S, Salvadori R, Bee L, Barbieri V, Mognato M. Analysis of DNA-damage response to ionizing radiation in serum-shock synchronized human fibroblasts. Cell Biol Toxicol. 2017;33(4):373-88. https://doi.org/10.1007/s10565-0179394-9.

Dai C, Li W, Tjong H, Hao S, Zhou Y, Li Q, et al. Mining 3D genome structure populations identifies major factors governing the stability of regulatory communities. Nat Commun. 2016;7:11549. https://doi.org/10.1038 /ncomms11549.

Dekker J, Belmont AS, Guttman M, Leshyk VO, Lis JT, Lomvardas S, et al. The 4D nucleome project. Nature. 2017;549(7671):219-26. https://doi.org/10.1038 /nature23884.
Dostie J, Blanchette M. 3D genome mapping and analysis methods. Methods. 2018;142:1-2. https://doi.org/10.1016/j. ymeth.2018.05.017.

Kim JH, Wang M, Lee J, Park HJ, Han C, Hong HS, et al. Prediction of hepatotoxicity for drugs using human pluripotent stem cell-derived hepatocytes. Cell Biol Toxicol. 2018;34(1):51-64. https://doi.org/10.1007/s10565-0179392-y.

Knoch TA, Wachsmuth M, Kepper N, Lesnussa M, Abuseiris A, Ali Imam AM, et al. The detailed 3D multi-loop aggregate/ rosette chromatin architecture and functional dynamic organization of the human and mouse genomes. Epigenetics Chromatin. 2016;9:58. https://doi.org/10.1186/s13072-0160089-X.

Li R, Liu Y, Hou Y, Gan J, Wu P, Li C. 3D genome and its disorganization in diseases. Cell Biol Toxicol. 2018. https://doi.org/10.1007/s10565-018-9430-4.

Ma T, Chen L, Shi M, Niu J, Zhang X, Yang X, et al. Developing novel methods to image and visualize 3D genomes. Cell Biol Toxicol. 2018. https://doi.org/10.1007/s10565-018-9427-z.

McLaren A. 1D->4D. Trends Genet. 1993;9(4):100.

Mizuguchi T, Barrowman J, Grewal SI. Chromosome domain architecture and dynamic organization of the fission yeast genome. FEBS Lett. 2015;589(20 Pt A):2975-86. https://doi. org/10.1016/j.febslet.2015.06.008.

Paulsen J, Gramstad O, Collas P. Manifold based optimization for single-cell 3D genome reconstruction. PLoS Comput Biol. 2015;11(8):e1004396. https://doi.org/10.1371/journal. pcbi. 1004396.

Quinodoz SA, Ollikainen N, Tabak B, Palla A, Schmidt JM, Detmar E, et al. Higher-order inter-chromosomal hubs shape 3D genome organization in the nucleus. Cell. 2018. https://doi.org/10.1016/j.cell.2018.05.024.

Szalaj P, Plewczynski D. Three-dimensional organization and dynamics of the genome. Cell Biol Toxicol. 2018. https://doi.org/10.1007/s10565-018-9428-y.

Terabayashi T, Hanada K. Genome instability syndromes caused by impaired DNA repair and aberrant DNA damage responses. Cell Biol Toxicol. 2018. https://doi.org/10.1007 /s10565-018-9429-x.

Tjong H, Li W, Kalhor R, Dai C, Hao S, Gong K, et al. Populationbased 3D genome structure analysis reveals driving forces in spatial genome organization. Proc Natl Acad Sci U S A. 2016;113(12):E1663-72. https://doi.org/10.1073 /pnas. 1512577113 .

Wang X. Clinical trans-omics: an integration of clinical phenomes with molecular multiomics. Cell Biol Toxicol. 2018;34(3): 163-6. https://doi.org/10.1007/s10565-018-9431-3.

Wang W, Zhu B, Wang X. Dynamic phenotypes: illustrating a single-cell odyssey. Cell Biol Toxicol. 2017;33(5):423-7. https://doi.org/10.1007/s10565-017-9400-2.

Wu P, Li T, Li R, Jia L, Zhu P, Liu Y, et al. 3D genome of multiple myeloma reveals spatial genome disorganization associated with copy number variations. Nat Commun. 2017;8(1):1937. https://doi.org/10.1038/s41467-017-01793-w.

Zeng Y, Chen X, Gao H, Wang X. An artificial intelligent single cell is part of the cell dream world. Cell Biol Toxicol. 2018;34:247-9. https://doi.org/10.1007/s10565-018-9433-1. 